\title{
Intra-gastric pH following single oral administrations of rabeprazole and esomeprazole: double-blind cross-over comparison
}

\author{
Kenji Furuta, ${ }^{1}$ Yukie Kohata, ${ }^{2}$ Yasuhiro Fujiwara, ${ }^{2}$ Mitsushige Sugimoto, ${ }^{3}$ Takahiro Uotani, ${ }^{3}$ Mihoko Yamade, ${ }^{3}$ \\ Shu Sahara, ${ }^{3}$ Hitomi Ichikawa, ${ }^{3}$ Takahisa Furuta, ${ }^{4}$ Kenta Nio, ${ }^{5}$ Ryuichi Iwakiri, ${ }^{5}$ Masahiko Inamori, ${ }^{6}$ \\ Osamu Kawamura, ${ }^{7}$ Motoyasu Kusano, ${ }^{8}$ Mototsugu Kato, ${ }^{9}$ Noriyuki Kawami, ${ }^{10}$ Katsuhiko Iwakiri, ${ }^{10}$ \\ Toshihisa Takeuchi, ${ }^{11}$ Kazuhide Higuchi, ${ }^{11}$ Masahito Aimi,, ${ }^{1 *}$ Kohji Naora, ${ }^{12}$ Kazuma Fujimoto, ${ }^{5}$ Tetsuo Arakawa ${ }^{2}$ \\ and Yoshikazu Kinoshita ${ }^{1}$ \\ 1Department of Gastroenterology and Hepatology and ${ }^{12}$ Department of Pharmacy, Shimane University Hospital, Shimane University School of Medicine \\ 89-1 Enya-cho, Izumo, Shimane 693-8501, Japan \\ 2Department of Gastroenterology, Osaka City University Graduate School of Medicine, 1-5-7 Asahi-cho, Abeno-ku, Osaka 545-8586, Japan \\ ${ }^{3}$ First Department of Medicine and ${ }^{4}$ Center for Clinical Research, Hamamatsu University School of Medicine, 1-20-1 Handayama, Higashi-ku, Hamamatsu, \\ Shizuoka 431-3192, Japan \\ ${ }^{5}$ Department of Internal Medicine, Saga Medical School, 5-1-1 Nabeshima, Saga 849-8501, Japan \\ ${ }^{6}$ Gastroenterology Division, Yokohama City University Hospital, 3-9 Fukuura, Kanazawa-ku, Yokohama, Kanagawa 236-0004, Japan \\ ${ }^{7}$ Department of Gastroenterology and ${ }^{8}$ Department of Endoscopy and Endoscopic Surgery, Gunma University Hospital, 3-39-15 Showa-cho, Maebashi, \\ Gunma 371-8511, Japan \\ ${ }^{9}$ Division of Endoscopy, Hokkaido University Hospital, Nishi 5-chome, Kita 14-jou, Kita-ku, Sapporo, Hokkaido 060-8648, Japan \\ ${ }^{10}$ Department of Gastroenterology, Nippon Medical School, Graduate School of Medicine, 1-1-5 Sendagi, Bunkyo-ku, Tokyo 113-8603, Japan \\ ${ }^{11}$ Second Department of Internal Medicine, Osaka Medical College, 2-7 Daigaku-cho, Takatsuki, Osaka 569-8686, Japan
}

(Received 28 February, 2014; Accepted 14 May, 2014; Published online 4 October, 2014)

\begin{abstract}
Comparisons between the acid inhibitory effects of rabeprazole and esomeprazole after single oral administration with standard doses have not been previously presented. We examined intragastric $\mathrm{pH}$ after oral administrations of these two proton pump inhibitors using 24-h pH monitoring. Fifty-four normal volunteers not infected by Helicobacter pylori were investigated. Using a cross-over design, we administered $10 \mathrm{mg}$ of rabeprazole or $\mathbf{2 0} \mathbf{~ m g}$ of esomeprazole in $\mathbf{2 7}$ at $\mathbf{3 0} \mathrm{min}$ after supper and in the remaining 27 subjects at 15 min before supper, and performed 24-h pH monitoring. Intra-gastric pH data were nearly identical when the proton pump inhibitors were taken after meals. Even if the data were compared in different CYP2C19 genotypes, rabeprazole and esomeprazole did not show the difference. In poor metabolizer, both of the drugs showed stronger acid inhibition. When taken before meals, intra-gastric pH after esomeprazole administration was slightly but not significantly higher than that observed after rabeprazole administration not only in daytime but also in nighttime period. In conclusion, rabeprazole and esomeprazole were similarly effective when administered after a meal.
\end{abstract}

Key Words: intra-gastric pH, rabeprazole, esomeprazole, double-blind, cross-over

D roton pump inhibitors (PPIs) potently inhibit gastric acid secretion, and are widely used for prevention and treatment of various acid-related diseases including peptic ulcers and gastroesophageal reflux diseases. Although their acid inhibitor potency is far stronger than that of histamine $\mathrm{H} 2$ receptor antagonists (H2RAs), PPIs are reported to have some weak points in comparison with those drugs. ${ }^{(1-3)}$

A disadvantage of the acid inhibitory effect of PPIs is the strong influence of CYP2C19, a hepatic drug metabolizing enzyme that degrades PPIs. ${ }^{(4)}$ In patients with high activity of the CYP2C19 enzyme (extensive metabolizers), the effect of PPI administration is not adequately strong, because of enzymatic degradation. On the other hand, in patients with a low CYP2C19 enzyme activity (poor metabolizers), the acid inhibiting effect of PPIs can be too strong. Another disadvantage is slow onset of the acid inhibitory effect after PPI administration. ${ }^{(1,2)}$

To improve these weak points, new types of PPIs have been developed and are widely used. Rabeprazole is a new type of PPI that is not strongly influenced by CYP2C19 enzyme activity, ${ }^{(4)}$ because it is not mainly degraded by CYP2C19.(5) In addition, this drug is reported to inhibit acid secretion more quickly than first generation PPIs including omeprazole and lansoprazole. ${ }^{(6)}$ Another agent is esomeprazole, an S-isomer of omeprazole that is a mixture of S- and R-isomers. Esomeprazole has also been reported to not be effectively degraded by CYP2C19 and its effect is not strongly influenced by its enzyme activity. ${ }^{(7-9)}$

The acid suppressing effects of rabeprazole $(20 \mathrm{mg})$ and esomeprazole $(40 \mathrm{mg})$ have been investigated, with those of the latter reported to be equal or superior to the former. ${ }^{(10-13)}$ The standard doses of rabeprazole and esomeprazole in Japan are 10 and $20 \mathrm{mg}$, respectively, per day. Those PPIs at those doses have not been directly compared in regard to quickness of acid inhibition and acid inhibitory potency in cases with different CYP2C19 enzyme activities. ${ }^{(14,15)}$ It is considered that intra-gastric $\mathrm{pH}$ monitoring soon after acute single administration of a PPI is an ideal experimental design to investigate its quick acid inhibitory effects.

In the present study, a single standard dose of rabeprazole or esomeprazole was administered to normal volunteers in a multicenter double-blind randomized prospective study with a crossover design and their effects on intra-gastric $\mathrm{pH}$ were compared.

\section{Materials and Methods}

Subjects. Fifty-seven healthy volunteers were enrolled at 9 university hospitals; Shimane University, Hokkaido University

*To whom correspondence should be addressed.

E-mail: aimi@med.shimane-u.ac.jp 
Table 1. Clinical characteristics of subjects

\begin{tabular}{lcc}
\hline & \multicolumn{2}{c}{ PPI administration } \\
\cline { 2 - 3 } & Before meal & After meal \\
\hline Number of cases & 27 & 27 \\
Male/female & $16 / 11$ & $15 / 12$ \\
Age (years) & $25.2 \pm 4.9^{*}$ & $23.6 \pm 3.0^{*}$ \\
Height $(\mathrm{cm})$ & $166.8 \pm 10.2^{*}$ & $168.3 \pm 7.5^{*}$ \\
Weight $(\mathrm{kg})$ & $58.4 \pm 10.7^{*}$ & $60.0 \pm 10.7^{*}$ \\
BMI & $20.8 \pm 2.2^{*}$ & $21.0 \pm 2.3^{*}$ \\
Alcoholic drink (+/-/+-) & $8 / 15 / 4$ & $4 / 19 / 4$ \\
Smoking (+/-/+-/?) & $1 / 20 / 4 / 2$ & $0 / 21 / 4 / 2$ \\
H. pylori (+/-) & $0 / 27$ & $0 / 27$ \\
CYP2C19 (IM/PM/RM) & $11 / 8 / 8$ & $17 / 5 / 5$ \\
\hline${ }^{*}$ mean + SD & &
\end{tabular}

Hospital, Gunma University Hospital, Nippon Medical School, Yokohama City University Hospital, Hamamatsu University School of Medicine, Osaka City University, Osaka Medical College, and Saga Medical School. The protocol utilized was approved by the institutional ethical committees of the participating institutions.

The clinical characteristics of the subjects are shown in Table 1. Their mean age was in the $20 \mathrm{~s}$ and none of the subjects was infected by Helicobacter pylori (H. pylori), which was determined by testing for the presence of the $H$. pylori antibody in serum and urine samples. The CYP2C19 genotype was tested by a polymerase chain reaction-restriction fragment length polymorphism (PCR-RFLP) assay, as previously reported..$^{(4)}$

pH monitoring. Twenty-nine of the 57 enrolled volunteers were randomly enrolled in the pre-meal PPI administration protocol. The subjects were investigated by $\mathrm{pH}$ monitoring twice, once with $10 \mathrm{mg}$ of rabeprazole and once with $20 \mathrm{mg}$ of esomeprazole. The PPIs were delivered in identical opaque gelatin capsules and discrimination between them was impossible during the study period. The identical opaque gelatin capsules containing $10 \mathrm{mg}$ rabeprazole or $20 \mathrm{mg}$ esomeprazole were prepared by an author pharmacist $(\mathrm{KN})$ and packaged in marked bags, and then delivered to each participating hospital. The key code of the drugs was kept by $\mathrm{KN}$ and opened firstly after fixing the final $\mathrm{pH}$ data. The order of administration was randomly determined for each subject. The $2 \mathrm{pH}$ monitoring examinations were separated by at least a 1-week interval. An intra-gastric $\mathrm{pH}$ monitoring sensor catheter (Zenetics Medical, Salt Lake City, UT) was introduced into the gastric body and monitoring was started at 17:00, as previously reported. ${ }^{(16,17)} \mathrm{A}$ single oral dose of the PPI was administered at $15 \mathrm{~min}$ before supper, at 18:45. The subjects started eating their supper (carbohydrates $112.8 \mathrm{~g}$, protein $16.3 \mathrm{~g}$, fat $27.3 \mathrm{~g}$, calories $762 \mathrm{kcal}$ ) at 19:00 and were asked to finish within $30 \mathrm{~min}$. The subjects were then requested to lie on their bed from 23:00 to 7:00 next morning. Breakfast (carbohydrates $34 \mathrm{~g}$, protein $5.8 \mathrm{~g}$, fat $2.8 \mathrm{~g}$, calories $85 \mathrm{kcal}$ ) and lunch (carbohydrates $74.4 \mathrm{~g}$, protein $17.1 \mathrm{~g}$, fat $11.4 \mathrm{~g}$, calories $531 \mathrm{kcal}$ ) were also consumed within $30 \mathrm{~min}$, starting at 8:00 and 12:00, respectively. Intra-gastric $\mathrm{pH}$ monitoring was terminated at 17:00. The remaining 28 subjects were enrolled in the postprandial PPI administration protocol group. All conditions were the same as above, except that rabeprazole or esomeprazole was administered orally $30 \mathrm{~min}$ after the end of supper at 20:00. In the postprandial administration protocol, the supper (carbohydrate $52.8 \mathrm{~g}$, protein $8.8 \mathrm{~g}$, fat $9.2 \mathrm{~g}$, calorie $330 \mathrm{kcal}$ ) and breakfast (carbohydrate $94 \mathrm{~g}$, protein $13.3 \mathrm{~g}$, fat $20.9 \mathrm{~g}$, calorie $617 \mathrm{kcal}$ ) were also slightly different from those used in the pre-meal administration protocol.

Median $\mathrm{pH}$ for each monitored hour and the percentage of time at which intra-gastric $\mathrm{pH}$ was below 4.0 were calculated for the total $24-\mathrm{h}$ period, as well as the daytime (7:00-23:00) and nighttime (23:00-7:00) periods.
Statistical analysis. Statistical analysis was performed using a Wilcoxon signed rank test when results of a Friedman test showed significant differences. The chronological data shown in Fig. 1, 3, 4 and 6 were analyzed by linear mixed models. A $p$ value of $<0.05$ was considered to be significant. The sample size of the study was calculated based on the previous studies comparing $40 \mathrm{mg}$ esomeprazole and $20 \mathrm{mg}$ rabeprazole on their first administration day. ${ }^{(10,12)}$ Hunfeld et al. ${ }^{(10)}$ calculated the number of necessary subjects as 18 based on parametric assumption and found the statistically significant results in their study. Warrington et al. ${ }^{(12)}$ enrolled 24 healthy subjects in their study. Therefore, in this study, 27 healthy subjects were enrolled in two different protocols (administration before or after a meal).

\section{Results}

Of the 57 enrolled subjects, 2 in the preprandial administration group and 1 in the postprandial group were not analyzed because of intolerance to the second $\mathrm{pH}$ monitoring examination. No adverse event occurred during $\mathrm{pH}$ monitoring. There were no significant differences in regard to gender, age, height, body weight, BMI, and CYP2C19 genotypes between the administration protocol groups (Table 1).

When administered before the meal, the median intra-gastric $\mathrm{pH}$ after esomeprazole administration tended to be higher than after rabeprazole administration (Fig. 1), whereas intra-gastric $\mathrm{pH}$ in the 2:00-3:00 time period was significantly higher after esomeprazole administration. At the other time points, there were no significant differences. We also calculated percent time of intra-gastric $\mathrm{pH}>4.0$ over the $24-\mathrm{h}$ period, as well as during the daytime and nighttime periods. Again, esomeprazole tended to show a stronger acid inhibitory effect, though differences with rabeprazole were not significant (Fig. 2). When the data was separately calculated for different CYP2C19 genotypes, esomeprazole raised intra-gastric $\mathrm{pH}$ more effectively in rapid metabolizers at 4 time points in 24-h observation period (Fig. 3), while there was no apparent difference between intra-gastric $\mathrm{pH}$ between rabeprazole and esomeprazole in the intermediate and poor metabolizers, except at a single time point in poor metabolizers.

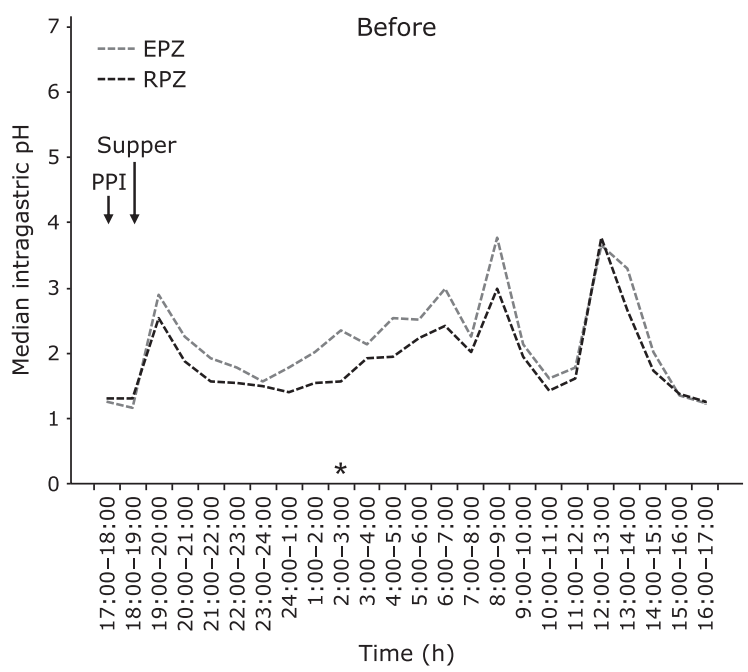

Fig. 1. Median intra-gastric $\mathrm{pH}$ for $24 \mathrm{~h}$ after a single preprandial oral administration of $10 \mathrm{mg}$ of rabeprazole (black line) or $20 \mathrm{mg}$ of esomeprazole (gray line). Using a cross-over design, $27 \mathrm{H}$. pylori uninfected subjects were studied with at least a 1-week interval between the rabeprazole and esomeprazole administrations. At only 1 time point measurement, esomeprazole raised intra-gastric $\mathrm{pH}$ to a significantly higher level than rabeprazole, while there were no significant differences found for the other time points. ${ }^{*} p<0.05$, statistically significant. 


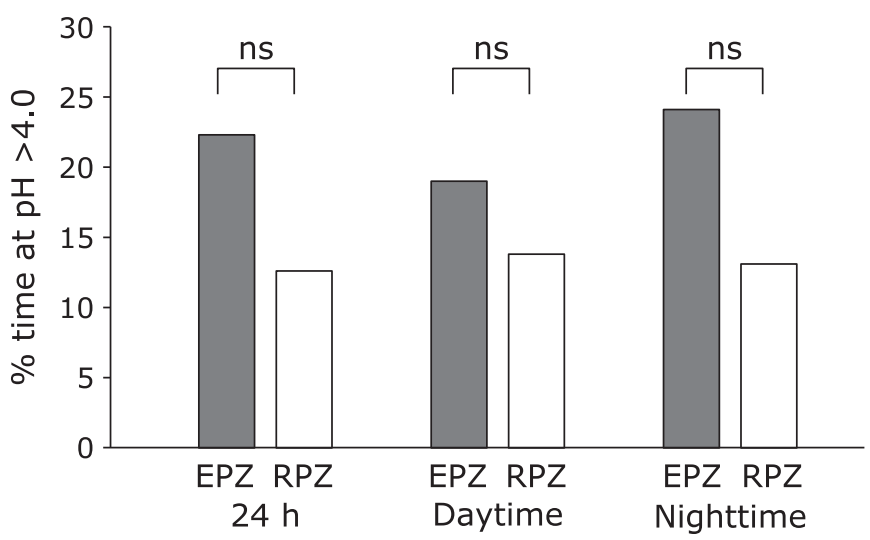

Fig. 2. Median \% time at $\mathrm{pH}>4.0$ during 24-h period after single preprandial oral administration of $10 \mathrm{mg}$ of rabeprazole (white column) or $20 \mathrm{mg}$ of esomeprazole (gray column) in 27 subjects. Although esomeprazole tended to elevate intra-gastric $\mathrm{pH}$ to a greater degree, there were no significant differences between the PPIs regarding acid inhibitory effects. RPZ, rabeprazole; EPZ, esomeprazole.

When the PPIs were administered after meals, there were no apparent differences in median intra-gastric $\mathrm{pH}$ at any time point after either administration (Fig. 4). Furthermore, after calculating percent time of intra-gastric $\mathrm{pH}>4.0$, there were no differences found during the daytime and nighttime periods (Fig. 5). Median intra-gastric $\mathrm{pH}$ was also calculated based on CYP2C19 genotype, and compared between rabeprazole and esomeprazole, with no significant difference found, except at a single time point in poor metabolizers (Fig. 6).

\section{Discussion}

The present results show that the acid inhibitory effects of $10 \mathrm{mg}$ of rabeprazole and $20 \mathrm{mg}$ of esomeprazole after single oral doses were similarly potent, especially when administered after meals. Four kinds of PPIs, omeprazole, lansoprazole, rabeprazole, and esomeprazole, are available for clinical practice in Japan, which can be divided into 2 groups based on their degradability by the hepatic drug metabolizing enzyme CYP2C19. ${ }^{(4,18,19)}$ Omeprazole and lansoprazole are easily degraded by CYP2C19, while rabeprazole and esomeprazole are not. Asian individuals are known to have heterogeneous CYP2C19 enzyme activity, as $30 \%$ are extensive metabolizers with high enzyme activity, $20 \%$ are poor metabolizers with low enzyme activity, and the remaining $50 \%$ are intermediate metabolizers. ${ }^{(20-22)}$ Therefore, different from western countries, the acid inhibitory effects of omeprazole and lansoprazole are known to be diverse among individuals. ${ }^{(4,19)}$ In cases with a high level of CYP2C19 enzyme activity, the acid inhibitory effects of these drugs are expected to be limited. To improve uncertainty, the more stable PPIs rabeprazole and esomeprazole are increasingly used in clinical practice for Japanese patients, with standard oral doses of 10 and $20 \mathrm{mg}$, respectively.

Rabeprazole is a newly developed racemic mixture compound reported to resist CYP2C19 degradation, ${ }^{(5)}$ while esomeprazole is an S-isomer of omeprazole and similarly resistant to CYP2C19. Therefore, these PPIs are considered to have a more consistent acid inhibitory effect irrespective of CYP2C19 enzyme activity. ${ }^{(7-9)}$ However, that of esomeprazole is considered to become submaximal when the drug is administered after a meal.

There are 2 possible mechanisms regarding this weak point of esomeprazole to consider, decreased absorption and incomplete activation. The plasma concentration of esomeprazole was investigated and compared when administered during fasting and after meals. ${ }^{(23,24)}$ Those results clarified that the plasma concentration of esomeprazole was higher when administered during fasting,
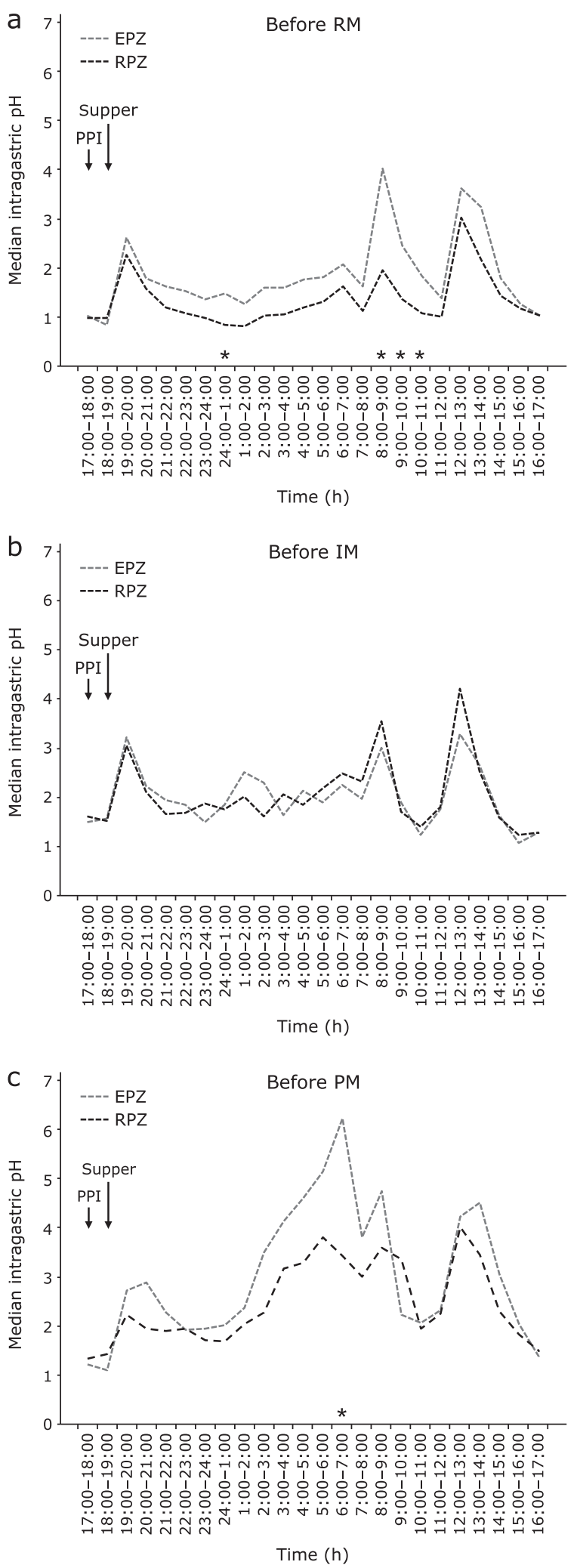

Fig. 3. Median intra-gastric $\mathrm{pH}$ during 24-h period after single preprandial oral administration of $10 \mathrm{mg}$ of rabeprazole (black lines) or $20 \mathrm{mg}$ of esomeprazole (gray lines) in (a) rapid metabolizers $(n=8)$, (b) intermediate metabolizers $(n=11)$, and (c) poor metabolizers $(n=8)$ of CYP2C19. In the rapid metabolizers, intra-gastric $\mathrm{pH}$ after administration of esomeprazole was significantly higher at 4 different time points as compared to rabeprazole. In intermediate and poor metabolizers, no significant and only one point significant differences were found, respectively. ${ }^{*} p<0.05$, statistically significant. 


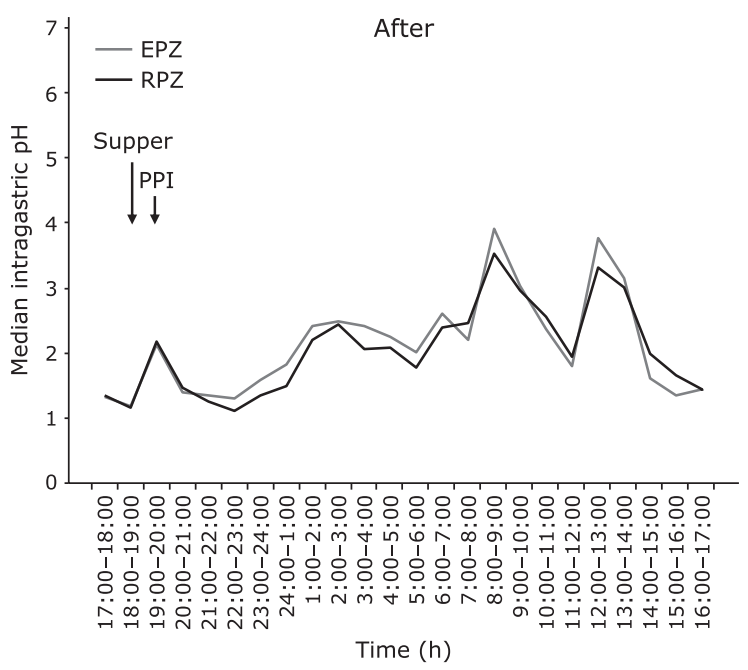

Fig. 4. Median intra-gastric $\mathrm{pH}$ during 24-h period after single postprandial oral administration of $10 \mathrm{mg}$ of rabeprazole (black line) or $20 \mathrm{mg}$ of esomeprazole (gray line). Using a cross-over design, $27 \mathrm{H}$. pylori uninfected subjects were studied with at least a 1-week interval between the rabeprazole and esomeprazole administrations. Intragastric $\mathrm{pH}$ values after administrations of rabeprazole and esomeprazole were nearly identical.

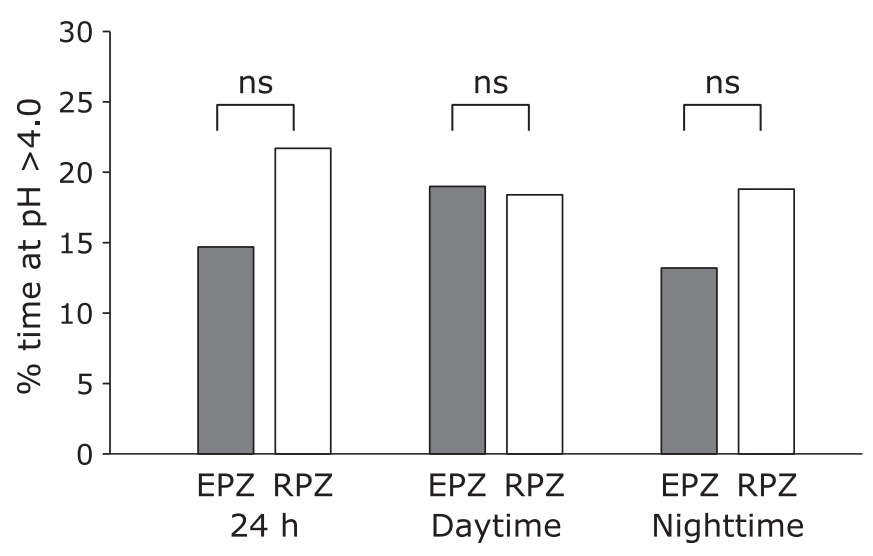

Fig. 5. Median \% time at $\mathrm{pH}>4.0$ during $24-\mathrm{h}$ period after single postprandial oral administration of $10 \mathrm{mg}$ of rabeprazole (white column) or $20 \mathrm{mg}$ of esomeprazole (gray column) in 27 subjects. There were no differences between esomeprazole and rabeprazole. RPZ, rabeprazole; $E P Z$, esomeprazole.

though the precise mechanism related to that difference is not clear. All PPIs need to be activated by the acidic environment in the secretory canaliculi of parietal cells. ${ }^{(25)}$ When administered after meals, an absorbed PPI will not be effectively activated because food-induced acid secretion and the highly acidic environment in the secretory canaliculi are nearly terminated when the plasma concentration of the drug reaches a peak level at 2-3 h after administration. Mainly based on data obtained from esomeprazole trials in western countries, PPIs are recommended to be administered 30 min before meals. ${ }^{(26-28)}$

On the other hand, the acid inhibitory effect of rabeprazole was shown to be not significantly influenced by timing of administration. In the present study, intra-gastric acidity after a single postprandial oral dose of rabeprazole $(10 \mathrm{mg})$ or esomeprazole $(20 \mathrm{mg})$ was similarly raised and remained nearly identical for $24 \mathrm{~h}$. On the other hand, the acid inhibitory effect of the latter was slightly stronger than that of the former when each was admin-
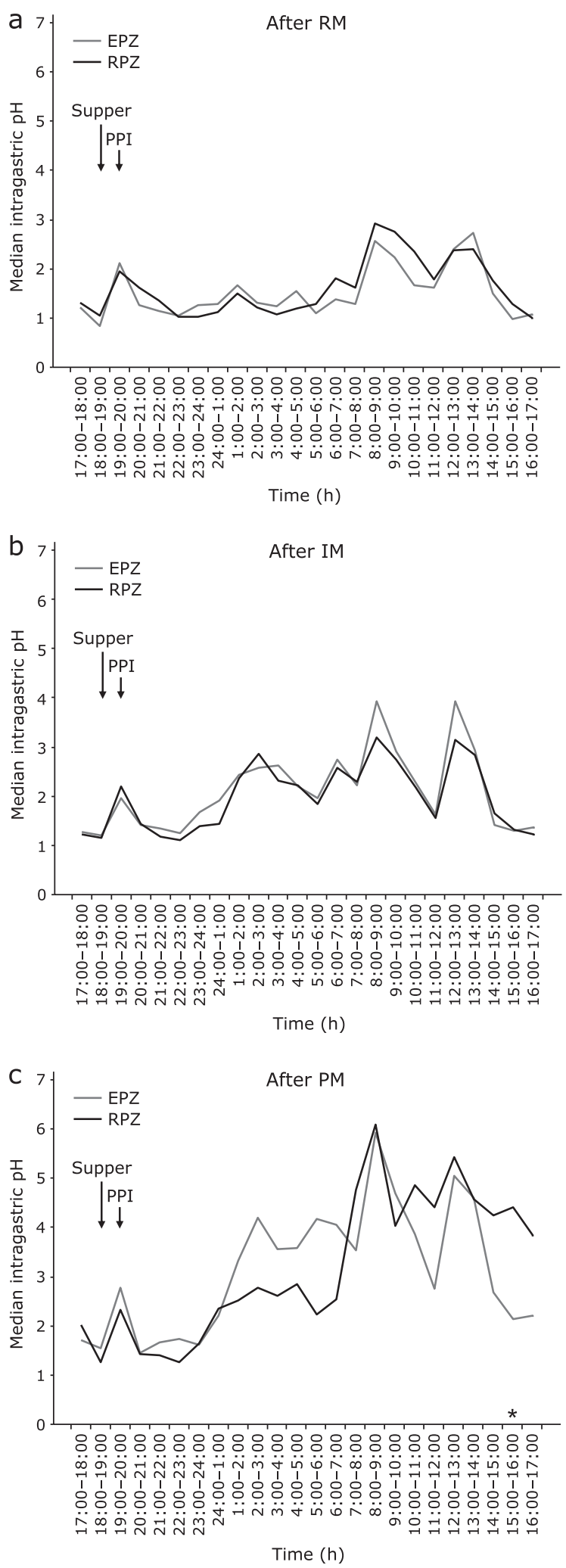

Fig. 6. Median intra-gastric $\mathrm{pH}$ during 24-h period after single postprandial oral administration of $10 \mathrm{mg}$ of rabeprazole (black lines) or $20 \mathrm{mg}$ of esomeprazole (gray lines) in (a) rapid metabolizers $(n=5)$, (b) intermediate metabolizers $(n=17)$, and (c) poor metabolizers $(n=5)$ of CYP2C19. At only 1 time point measurement in poor metabolizers, rabeprazole raised intra-gastric $\mathrm{pH}$ to a significantly higher level than esomeprazole, while there were no significant differences found for the other time points. ${ }^{*} p<0.05$, statistically significant. 
istered before meals, though the difference was not statistically significant. These results confirm a previous report showing that esomeprazole had a stronger acid inhibitory effect when administered $30 \mathrm{~min}$ before meals.

In the present study, direct comparisons of the acid inhibitory effects of the tested PPIs between pre- and post-prandial administrations was difficult, since the foods taken during the monitoring periods were not identical. However, when we compared the pre- and post-prandial administrations, esomeprazole was stronger with pre-prandial administration, as previously reported, while rabeprazole was equally potent irrespective of the timing of administration.

In Japan, approximately $80 \%$ of physicians instruct their patients to take PPIs after breakfast and approximately $10 \%$ after dinner. ${ }^{(29)}$ Therefore, $90 \%$ of the patients take PPIs after meals. In such an environment, the acid inhibitory effects of the present PPI administrations are considered to be nearly identical, though esomeprazole may show a statistically non-significant benefit when administered before meals.

There are some limitations to our study. The first is lack of baseline intra-gastric $\mathrm{pH}$ data obtained without any drug administration. To more sensitively check the potency of any acid inhibitory effect, baseline $\mathrm{pH}$ data are necessary. Therefore, a comparison of the intra-gastric $\mathrm{pH}$ observed after rabeprazole and esomeprazole administrations is the only one possible in this study. Secondly, we did not measure the plasma PPI levels in subjects. Therefore, we could not correlate the pharmacokinetic disposition of PPI with the intragastric $\mathrm{pH}$. The influence of meal on the absorption of PPIs and their acid inhibitory effects could not be made clear. Another is the lack of $\mathrm{pH}$ data during chronic administration of the PPIs, since these drugs are frequently used for chronic treatment. An additional study with chronic administrations of PPIs as well as baseline data may be necessary in the future.

\section{References}

1 Khoury RM, Katz PO, Castell DO. Post-prandial ranitidine is superior to post-prandial omeprazole in control of gastric acidity in healthy volunteers. Aliment Pharmacol Ther 1999; 13: 1211-1214.

2 Inamori M, Togawa J, Iwasaki T, et al. Early effects of lafutidine or rabeprazole on intragastric acidity: which drug is more suitable for on-demand use? J Gastroenterol 2005; 40: 453-458.

3 Katsube T, Adachi K, Kawamura A, et al. Helicobacter pylori infection influences nocturnal gastric acid breakthrough. Aliment Pharmacol Ther 2000; 14: 1049-1056.

4 Adachi K, Katsube T, Kawamura A, et al. CYP2C19 genotype status and intragastric $\mathrm{pH}$ during dosing with lansoprazole or rabeprazole. Aliment Pharmacol Ther 2000; 14: 1259-1266.

5 Ishizaki T, Horai Y. Review article: cytochrome P450 and the metabolism of proton pump inhibitors--emphasis on rabeprazole. Aliment Pharmacol Ther 1999; 13(Suppl 3): 27-36.

6 Saitoh T, Fukushima Y, Otsuka H, et al. Effects of rabeprazole, lansoprazole and omeprazole on intragastric $\mathrm{pH}$ in CYP2C19 extensive metabolizers. Aliment Pharmacol Ther 2002; 16: 1811-1817.

7 Andersson T, Bredberg E, Sunzel M, Antonsson M, Weidolf L. Pharmacokinetics (PK) and effect on pentagastrin stimulated peak acid output (PAO) of omeprazole $(\mathrm{O})$ and its 2 optical isomers, S-omeprazole/esomeprazole (E) and R-omeprazole (R-O). Gastroenterology 2000; 118(4 Pt II): A1210.

8 Andersson T, Hassan-Alin M, Hasselgren G, Röhss K, Weidolf L. Pharmacokinetic studies with esomeprazole, the (S)-isomer of omeprazole. Clin Pharmacokinet 2001; 40: 411-426.

9 Hassan-Alin M, Andersson T, Niazi M, Röhss K. A pharmacokinetic study comparing single and repeated oral doses of $20 \mathrm{mg}$ and $40 \mathrm{mg}$ omeprazole and its two optical isomers, S-omeprazole (esomeprazole) and R-omeprazole, in healthy subjects. Eur J Clin Pharmacol 2005; 60: 779-784.

10 Hunfeld NG, Touw DJ, Mathot RA, van Schaik RH, Kuipers EJ. A comparison of the acid-inhibitory effects of esomeprazole and rabeprazole in relation to pharmacokinetics and CYP2C19 polymorphism. Aliment Pharmacol Ther
In summary, we found that the intra-gastric $\mathrm{pH}$ values for $24 \mathrm{~h}$ after a single oral dose of rabeprazole $(10 \mathrm{mg})$ or esomeprazole $(20 \mathrm{mg})$ were nearly identical, especially when administered after meals. On the other hand, preprandial administration of esomeprazole may slightly augment its acid inhibitory effect.

\section{Conflicts of Interest}

Furuta $\mathrm{K}$ received research grant from AstraZeneca KK, Eisai Co., Ltd. and Daiichi-Sankyo Co., Ltd. Fujiwara Y received lecture fee from Eisai Co., Ltd. The Center for Clinical Research and the First Department of Medicine at Hamamatsu University School of Medicine have received grants from Takeda Pharmaceutical Co., Ltd., AstraZeneca KK, Eisai Co., Ltd., DaiichiSankyo Co., Ltd., and Sugimoto $\mathrm{M}$ and Furuta $\mathrm{T}$ have received lecture fees from Takeda Pharmaceutical Co., Ltd., AstraZeneca KK, Eisai Co., Ltd., Daiichi-Sankyo Co., Ltd. Kusano M received lecture fee and research grant from Eisai Co., Ltd., and lecture fee from AstraZeneca KK and Daiichi-Sankyo Co., Ltd. Kato M received lecture fees from Eisai Co., Ltd., Takeda Pharmaceutical Co., Ltd., and AstraZeneca KK and received research funds from Eisai Co., Ltd., Takeda Pharmaceutical Co., Ltd., Otsuka Pharmaceutical Co., Ltd., AstraZeneca KK, Astellas Pharmaceutical Co., Ltd., and Daiichi-Sankyo Co., Ltd. Iwakiri K received lecture fee from Eisai Co., Ltd. Higuchi $\mathrm{H}$ and Fujimoto $\mathrm{K}$ received research grant and lecture fees from AstraZeneca KK, Eisai Co., Ltd., Daiichi-Sankyo Co., Ltd. Naora K received research grants from AstraZeneca KK, Eisai Co., Ltd., and Takeda Pharmaceutical Co., Ltd. Arakawa T received research grant from Eisai Co., Ltd. and Otsuka Pharm Co., and lecture fee from Eisai Co., Ltd. Kinoshita Y received research grants and lecture fees from AstraZeneca KK, Eisai Co., Ltd., Daiichi-Sankyo Co., Ltd.

This study was funded by Eisai Co., Ltd.
2012; 35: 810-818.

11 Wilder-Smith CH, Röhss K, Nilsson-Pieschl C, Junghard O, Nyman L. Esomeprazole $40 \mathrm{mg}$ provides improved intragastric acid control as compared with lansoprazole $30 \mathrm{mg}$ and rabeprazole $20 \mathrm{mg}$ in healthy volunteers. Digestion 2003; 68: 184-188.

12 Warrington S, Baisley K, Dunn K, Boyce M, Morocutti A. Effects of single doses of rabeprazole $20 \mathrm{mg}$ and esomeprazole $40 \mathrm{mg}$ on 24-h intragastric $\mathrm{pH}$ in healthy subjects. Eur J Clin Pharmacol 2006; 62: 685-691.

13 Miner P Jr, Katz PO, Chen Y, Sostek M. Gastric acid control with esomeprazole, lansoprazole, omeprazole, pantoprazole, and rabeprazole: a five-way crossover study. Am J Gastroenterol 2003; 98: 2616-2620.

14 Norris V, Baisley K, Dunn K, Warrington S, Morocutti A. Combined analysis of three crossover clinical pharmacology studies of effects of rabeprazole and esomeprazole on 24-h intragastric $\mathrm{pH}$ in healthy volunteers. Aliment Pharmacol Ther 2007; 25: 501-510.

15 Röhss K, Wilder-Smith C, Nauclér E, Jansson L. Esomeprazole 20mg provides more effective intragastric Acid control than maintenance-dose rabeprazole, lansoprazole or pantoprazole in healthy volunteers. Clin Drug Investig 2004; $24: 1-7$.

16 Adachi K, Komazawa Y, Mihara T, et al. Comparative study of the speed of acid-suppressing effects of oral administration of cimetidine and famotidine. J Gastroenterol Hepatol 2005; 20: 1012-1015.

17 Komazawa Y, Adachi K, Mihara T, et al. Tolerance to famotidine and ranitidine treatment after 14 days of administration in healthy subjects without Helicobacter pylori infection. J Gastroenterol Hepatol 2003; 18: 678-682.

18 Yasuda S, Horai Y, Tomono Y, et al. Comparison of the kinetic disposition and metabolism of E3810, a new proton pump inhibitor, and omeprazole in relation to S-mephenytoin 4'-hydroxylation status. Clin Pharmacol Ther 1995; 58: 143-154.

19 Shirai N, Furuta T, Moriyama Y, et al. Effects of CYP2C19 genotypic differences in the metabolism of omeprazole and rabeprazole on intragastric 
pH. Aliment Pharmacol Ther 2001; 15: 1929-1937.

20 Kubota T, Chiba K, Ishizaki T. Genotyping of S-mephenytoin 4'-hydroxylation in an extended Japanese population. Clin Pharmacol Ther 1996; 60: 661-666.

21 Nakamura K, Goto F, Ray WA, et al. Interethnic differences in genetic polymorphism of debrisoquin and mephenytoin hydroxylation between Japanese and Caucasian populations. Clin Pharmacol Ther 1985; 38: 402408.

22 Horai Y, Nakano M, Ishizaki T, et al. Metoprolol and mephenytoin oxidation polymorphisms in Far Eastern Oriental subjects: Japanese versus mainland Chinese. Clin Pharmacol Ther 1989; 46: 198-207.

23 Junghard O, Hassan-Alin M, Hasselgren G. The effect of the area under the plasma concentration vs time curve and the maximum plasma concentration of esomeprazole on intragastric pH. Eur J Clin Pharmacol 2002; 58: 453-458.

24 Sostek MB, Chen Y, Andersson T. Effect of timing of dosing in relation to food intake on the pharmacokinetics of esomeprazole. Br J Clin Pharmacol 2007; 64: 386-390.

25 Shin JM, Sachs G. Pharmacology of proton pump inhibitors. Curr Gastroenterol Rep 2008; 10: 528-534.

26 Hershcovici T, Fass R. Management of gastroesophageal reflux disease that does not respond well to proton pump inhibitors. Curr Opin Gastroenterol 2010; 26: 367-378.

27 Hershcovici T, Fass R. Step-by-step management of refractory gastresophageal reflux disease. Dis Esophagus 2013; 26: 27-36.

28 Katz PO, Gerson LB, Vela MF. Guidelines for the diagnosis and management of gastroesophageal reflux disease. Am J Gastroenterol 2013; 108: 308328 .

29 Koshino K, Miki M, Azumi T, Furuta K, Adachi K, Kinoshita Y. Is the timing of PPI doseing appropriate in Japan: research on gastroenterologists. Clin Gastroenterol 2007; 10: 109-113. 